\title{
INCIDENCE OF ASSOCIATED TRAUMATIC INJURIES AND COMORBIDITIES IN RELATION TO LONG BONE FRACTURES IN CHILDREN - PROSPECTIVE ANALYSIS OF 291 CASES
}

\author{
Maria Daniela Trăilescu ${ }^{1,2}$, Adrian Ionel Pavel ${ }^{1,2}$, Alexandru Mircea Pop ${ }^{1}$
}

\section{Abstract}

Background and objectives: The study's objective was to investigate the incidence of associated traumatic injuries and comorbidities in relation to long bone fractures among children aged 5-18 years. Materials and Methods: The study population consists of children aged 5-18 years and included 291 patients with long bone fractures hospitalized and operated in a single institution, over the period 20152018. Results: Our results show an increased incidence of traumatic injuries associated with long bone fractures in children, as well as an increased prevalence of comorbidities. Compared to the number of long bone fractures, the associated injuries were found in $85.22 \%$ of cases. Compared to the number of patients studied, the percentage of comorbidities in the group studied is $24.74 \%$. Iron deficiency anemia, obesity and ADHD syndrome, sequelae of rickets and insulin-dependent diabetes mellitus were the most common comorbidities. Conclusions: The myth that the fracture in children occurs in a healthy field, without comorbidities, raises questions in the case of these concrete data. Thus, it is necessary to diagnose the injuries associated with fractures in children, as well as comorbidities by correct historical clinical and paraclinical examinations that are extremely important in the preoperative evaluation of the patient and in the subsequent evolution towards healing.

Keywords: children; fractures; injuries; comorbidities

\section{Introduction}

Commonly encountered in pediatric traumatic pathology, the incidence of fractures in children has become high in the conditions of modern life, where road accidents, sports and play occupy the first place and are of particular interest to children.

It is realistic to accept that "all children are sometimes prone to accidents" as Gusstafson states [1] and as medical practice demonstrates daily. Pediatric fractures account for approximately $25 \%$ of all pediatric traumatic injuries [2,3]. According to a recent report, trauma is the 28th leading cause of death globally [4].

Various studies have identified the young population as the most vulnerable group affected by fall-related injuries, with boys being affected twice as often as girls.

Falling from a height has also been recognized as one of the most common mechanisms of trauma in low-income countries [5].

Some studies report that the risk of having a fracture during childhood is almost $50 \%$ for boys and $30 \%$ for girls [6-14]. Recent studies [15-17] have evaluated and established that attention deficit hyperactivity disorder (ADHD) is a risk factor for fractures in children with this condition in which the daily condition is permanently characterized by attention deficit and inadequate hyperactivity or impulsivity, which affects all aspects of social life.

Our study aims to investigate the prevalence of associated traumatic injuries and comorbidities in relation to long bone fractures among children aged 5-18 years hospitalized in our department which required surgery to stabilize the long bone fractures.

\section{Material and method}

This is a prospective study that aims to analyze and investigate the prevalence of associated injuries and comorbidities in relation to long bone fractures among children aged 5-18 years. The study population consists of patients with long bone fractures hospitalized and operated in a single institution.

The study was conducted in accordance with the ethical principles from the Declaration of Helsinki and it respects ethical demands that are required by research with human subjects. Before starting the study, approval from the Human Research and Ethics Committee of the "Vasile Goldiș" Western University of Arad and from the Emergency County Hospital, Arad, Romania, was obtained (number of approval 2/19.07.2018 and 128/7.12.2018).

The study was carried out at the Pediatric Surgery and Orthopedics Department, Emergency County Hospital, Arad, Romania, between 2015-2018, and included 291 children with long bone fractures hospitalized for surgical treatment.

All patients and/or parents of patients were interviewed for personal medical history of the child, family history, the context of traumatic injury. In addition, imaging scans focusing on obvious traumatic injuries were performed to establish the definite diagnosis of traumatic injuries, supplemented by laboratory tests.

${ }^{1}$ Department of Pediatric Surgery and Orthopedics, Emergency County Hospital, Arad, Romania

${ }^{2}$ Faculty of Medicine, "Vasile Goldiş" Western University of Arad, Romania

E-mail: trailescumaria@yahoo.com, adipavel72@yahoo.com, alexanpop@yahoo.com 
For all the cases studied we used coding related to fractures, classified by ICD-10 (The International Classification of Diseases - The tenth Revision), of humerus (S42.2 - S42.4, S42.7), femur (S72.0 - S72.9), radius and /or ulna (S52.0 - S52.9) and tibia and /or fibula (S82.1-S82.9). For all associated injuries and/or comorbidities we used the same coding classified by ICD10 .

\subsection{Identification of the type of fracture}

X-rays of the limb with clinical signs of fracture, anteroposterior and lateral views, were performed in all patients studied. Based on the radiological examination, the type of fracture and the indication for treatment were established. Radiography of other anatomical regions, as well as ultrasound or CT examination were indicated in polytrauma patients. The obtained results allowed the identification of lesions associated with fractures of long bones in children.

\subsection{Laboratory investigations}

The preoperative biological balance for all cases with surgical indication complied with the protocol adopted at the hospital level: complete blood count, urea, creatinine, blood glucose, transaminases, urine examination. In patients with associated traumatic injuries or in polytrauma patients, the biological balance has been extended, including blood group, Rh, bleeding time (BT), clotting time (CT), prothrombin time (PT), activated thromboplastin time (aPTT) and thrombin time (TT).

\section{Results}

Clinical examination of patients with diaphyseal fractures revealed clinical signs of probability in all patients, while clinical signs of certainty were identified in only 251 cases. In 40 cases, the externalization of the fractured fragment by the solution of skin continuity established the definite diagnosis of open fracture and made it unnecessary to perform local examination maneuvers, which would have accentuated the suffering of young patients. From a radiological point of view, the diagnosis of diaphyseal fractures was made in $100 \%$ of cases, without the need for further investigations.

The distribution of patients among gender, different age and count of associated injuries and comorbidities is presented in Table 1.

In polytrauma patients, the clinical examination identified in addition to certain clinical signs of fracture and signs of cerebral distress in 65 cases (drowsiness, amnesia, vomiting, headache), as well as signs of hemorrhagic shock in 49 cases, in which the diagnostic strategy was focused on the major lesion (hepatic rupture, splenic rupture), as well as the therapeutic conduct (Figure 1).

Compared to the total number of diaphyseal fractures, the associated injuries were found in $85.22 \%$ of cases.

Figure 1 shows the traumatic injuries associated with patients with long bone fractures at the time of admission in hospital.

It can be observed that the associated minor injuries, represented by wounds, hematomas and contusions, were the most common traumatic injuries associated with diaphyseal fractures in 97 cases accounting for 39\% of injuries.

Closed acute craniocerebral injuries that required pediatric neurological consultation and specific medication accounted for $26 \%$ of injuries, intraabdominal parenchymal organ injuries that required specific treatment accounted for $20 \%$ of injuries, while other types of fractures associated with diaphysis fracture accounted for $15 \%$ of injuries, followed in frequency.

The coexistence of major traumatic injuries required the stabilization of the patient by medical or surgical therapeutic methods, the stabilization of fractures being performed in a secondary time. Monitoring of patients in the pediatric intensive care unit was essential, the operative moment being established by agreement with the anesthetist.

Out of the total number of operated patients, 72 cases presented comorbidities (Figure 2).

\begin{tabular}{ccccc}
\hline Study population & $\begin{array}{c}\text { Patients } \\
\text { (no.) }\end{array}$ & $\begin{array}{c}\text { Age } \\
\text { (yrs.) }\end{array}$ & $\begin{array}{c}\text { Count of associated } \\
\text { injuries } \\
\text { (\%) }\end{array}$ & $\begin{array}{c}\text { Count of comorbidities } \\
\text { (\%) }\end{array}$ \\
\hline $\begin{array}{c}\text { Long bone } \\
\text { fractures } \\
\text { in children }\end{array}$ & & & $85.22 \%$ & $24.74 \%$ \\
Female & 91 & 12 & \\
Male & 200 & $15,17)$ & \\
& & $(5,18)$ &
\end{tabular}

Data for distribution of patients among gender, different age and count of associated injuries and comorbidities. The patients' age is shown as median values and minimum and maximum values (in parenthesis).

Table 1. The measured values of count of associated injuries and comorbidities in relation to long bone fractures in children and according to gender. 


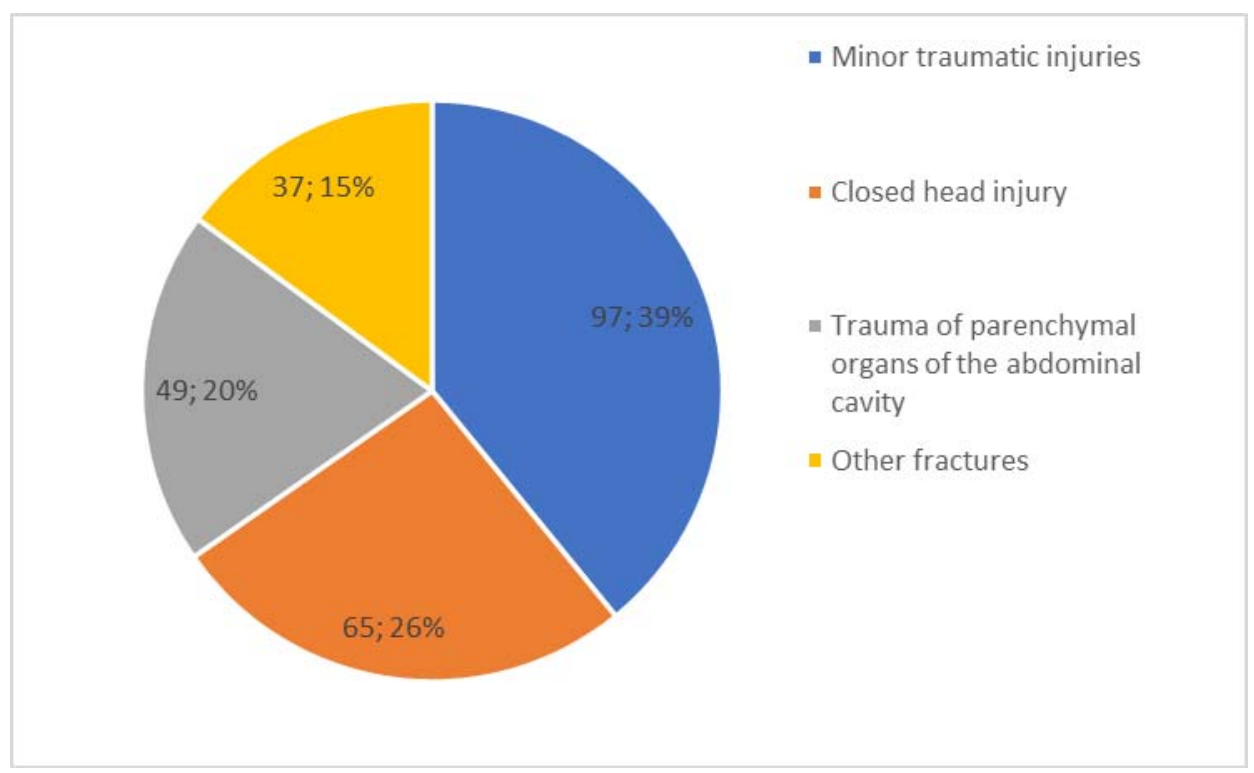

Fig. 1. Associated injuries in relation to long bone fracture in children.

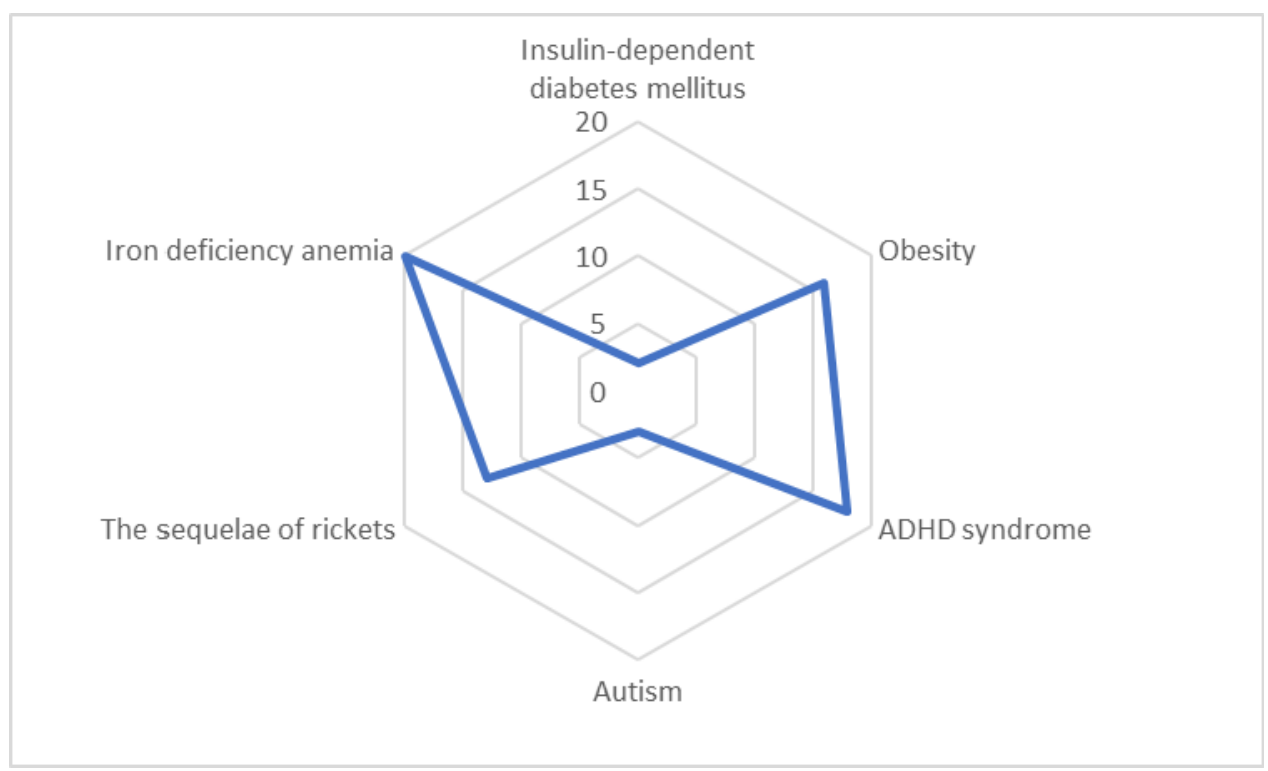

Fig. 2. Associated comorbidities in relation to long bone fracture in children according to the study group. 
Compared to the number of operated patients, the percentage of comorbidities in the group studied is $24.74 \%$. Iron deficiency anemia, obesity and ADHD syndrome were the most common comorbidities. Thus, the myth by which the fracture in children occurs in a healthy, untreated ground, raises questions in the case of these concrete data.

Compared to the number of operated patients, the percentage of comorbidities in the group studied is $24.74 \%$. Iron deficiency anemia, obesity and ADHD syndrome were the most common comorbidities. Thus, the myth by which the fracture in children occurs in a healthy, untreated ground, raises questions in the case of these concrete data.

\section{Discussion}

To our knowledge this study examined for the first time in Romania the prevalence of associated traumatic injuries and comorbidities in relation to long bone fractures in children. Our research results expand the knowledge about the associated injuries and comorbidities of fractures in children and offer new data regarding correct diagnosis approach in pediatric patients with long bone fractures. Our study focused on the determination of associated injuries and comorbidities in children with long bone fractures.

Although our study focuses on the young body, with the peculiarities of the musculoskeletal system, the frequency and complexity of fractures in children is a global problem, especially when recent studies show that $25 \%$ of traumatic pathology in children are fractures $[6,18$, $19]$.

It is well known that, in pediatric traumatology, conservative orthopedic treatment allows certain degrees of angulation or displacement, permissiveness due to the physiological process of accentuated bone remodeling after indirect healing with initial hypertrophic callus. If we omit the unique ability of immature bone to reshape and correct displacements or angles described, demonstrated and accepted by pediatric orthopedists, osteosynthesis can be performed on any type of fracture in a comprehensive manner, with disastrous results.

The increased prevalence of cases with indication for orthopedic surgical treatment in our department demonstrates complex traumatic mechanisms of production, resulting in complex fractures of long bones in children and multiple associated traumatic injuries.

The incidence of traumatic pathology by sex groups, respectively $69 \%$ male and $31 \%$ female, corresponds to the data in the literature and is explained by the concern and involvement of males in extreme sports and physical activities.

Clinical and paraclinical investigations demonstrated that the long bone fractures in children highlight two important aspects that should be considered in the future by every pediatric orthopedist.
Firstly, the diagnosis of injuries associated with long bone fractures is mandatory, in the case of polytraumas taking priority patient stabilization and treatment of organ injuries, with surgical stabilization of fractures being performed later. In our study, monitoring of patients in the pediatric intensive care unit was essential in $20 \%$ of cases which demonstrates the association of major traumatic injuries (traumatic intra-abdominal injuries) associated with fractures.

Secondly, the diagnosis of comorbidities requires the establishment of appropriate treatment after surgical stabilization of fractures [20].

Compared to the number of operated patients, the percentage of comorbidities in the groups studied is $24.74 \%$. The anatomical healing of the fracture in the child is directly dependent on the orthopedic treatment, but the coexistence of some undiagnosed pathologies can adversely influence the healing. Thus, it is necessary to diagnose comorbidities in close collaboration with pediatricians.

Our paper has some weaknesses. Firstly, the observation is made over a relatively short period of time in a single hospital and includes patients with long bone fractures that require surgery. In this situation, we do not have a study of associated lesions or comorbidities in patients with long bone fractures who were treated on an outpatient basis, in which case, the percentage of associated traumatic injuries and comorbidities could have been higher.

This paper also has several strengths. First of all, our study includes a significant number of children with long bone fractures. Secondly, the medical care of children with fractures is provided within a centralized system, involving a limited number of qualified physicians. This gives credibility to the diagnosis, on the one hand, and makes the collection of information quite easy and complete, on the other hand. Finally, the methodology used to collect information has been the same for over 10 years, namely direct contact with the pediatric orthopedic surgeon managing these children, being well developed and contributing to the reliability of the information.

\section{Conclusions}

Our data demonstrated an alarming incidence of associated traumatic injuries and comorbidities in long bone fractures in children. To confirm this, however, collecting information from larger populations from different geographical regions and monitoring the incidence over a period of several years are needed.

Conflicts of Interest: The authors declare no conflict of interest

\section{References}

1. Gustafsson LH. Childhood accidents. Three epidemiological studies on the etiology. Scand. J. SOC. Med. 1977; 5, 5-13.
2. Rennie L, Court-Brown CM, Mok JYQ et al. The epidemiology of fractures in children. Injur. 2007; 38:913-22. 
3. Kopjar B, Wickizer T. Fractures among children: incidence and impact on daily activities. Inj Prev. 1998; 4:194-7.

4. Rodà D, Trenchs V et al. Epidemiology of Fractures in Children Younger Than 12 Months. Pediatric Emergency Care. 2019; 35 (4) 256-260. DOI: 10.1097/PEC.0000000000001157.

5. Lyons RA, Delahunty AM, Kraus D, Heaven M, McCabe M, Allen H, Nash P. Children's fractures: a populationbased study. Inj Prev; 1999, 5:129-32.

6. Lyons RA, Sellstrom E, Delahunty AM, Loeb M, Varilo S. Incidence and cause of fractures in European districts. Arch Dis Child. 2000; 82:452-5.

7. Mäyränpää $M K$, Mäkitie $O$, Kallio PE. Decreasing incidence and changing pattern of childhood fractures: A population-based study. J Bone Miner Res. 2010; 25(12):2752-9.

8. Landin LA. Fracture patterns in children. Analysis of 8,682 fractures with special reference to incidence, etiology and secular changes in a Swedish urban population 1950-1979. Acta Orthop Scand. 1983; 202: 1-109.

9. Landin LA, Nilsson BE. Bone mineral content in children with fractures. Clin Orthop Relat Res. 1983; vol. 178, 292-296.

10. Landin LA. Epidemiology of children's fractures. J Pediatr Orthop B. 1997; vol. 6, no. 2,79-83.

11. Spady D W, Saunders D L, Schopflocher D P, Svenson L. W. Patterns of injury in children: a population-based approach. Pediatrics. 2004; vol. 113, part. 1, no. 3, 522-529,

12. Cheng JC, Ng BK, Ying SY, Lam PK. A 10-year study of the changes in the pattern and treatment of 6,493 fractures. J Pediatr Orthop. 1999; vol. 19, no. 3, 344350 .
13. Cooper C, Dennison EM, Leufkens HG, Bishop N, van Staa TP. Epidemiology of childhood fractures in Britain: a study using the general practice research database. J Bone Miner Res. 2004; vol. 19, no. 12, 1976-1981.

14. van Staa TP, Dennison EM, Leufkens HG, Cooper C. Epidemiology of fractures in England and Wales. Bone. 2001; vol. 29, no. 6, 517-522.

15. Uslu MM, Uslu R. Extremity fracture characteristics in children with impulsive/hyperactive behavior. Arch Orthop Trauma Surg. 2008; 128(4):417-21.

16. Shilon Y, Pollak Y, Aran A, Shaked S, Gross-Tsur V. Accidental injuries are more common in children with attention deficit hyperactivity disorder compared with their non-affected siblings. Child Care Health Dev. 2012; 38(3):366-70.

17. Lam LT. Attention deficit disorder and hospitalization owing to intra- and interpersonal violence among children and young adolescents. J Adolesc Health. 2005; 36:19-24.

18. Brudvik C, Hove LM. Childhood fractures in Bergen Norway: Identifying High-risk Groups and Activities. J Pediatr Orthop. 2003; 23: 629-634.

19. Guice KS, Cassidy LD, Oldham KT Traumatic injury and children: a national assessment. J Trauma. 2007; vol. 63, 68-80.

20. Torres L, Lycans D, Raducan V. Principles of Pediatric Fracture Treatment. Orthopedic Surgery Clerkship. 2007; 529-534.

Correspondence to:

\section{Maria Daniela Trăilescu}

Department of Pediatric Surgery and Orthopedics,

Emergency County Hospital, Arad, Romania

Andreny Karolly Street, No.2 - 4,

Arad, 310037, România

Phone: +40-257- 220000

Email: trailescumaria@yahoo.com 